\title{
Seeweite Bodensee-Messkampagne 2007 als Test für BodenseeOnline
}

\author{
Zwischen dem 22. April und dem 16. Mai 2007 veranstaltete das Kooperations-Netzwerk \\ Bodensee (KNB) eine seeweite Messkampagne zur Ermittlung der 3-D-Verteilung von Tem- \\ peratur, Phyto- und Zooplankton und deren kurzzeitiger Dynamik. Dabei konnten deutliche \\ horizontale Gradienten nachgewiesen werden, die während eines Starkwindereignisses \\ von der Hydrodynamik bestimmt wurden. Parallel dazu wurden mit dem gekoppelt hydro- \\ dynamisch-ökologischen Modell ELCOM-CAEDYM Modellrechnungen durchgeführt.
}

\section{Motivation und Organisation}

Das Gewässermonitoring des Instituts für Seenforschung und der Universität Konstanz am Bodensee liefert umfassende Zeitreihen zu Messwerten verschiedener biotischer und abiotischer Parameter im See, allerdings nur an wenigen Messstellen, die keine horizontale Diskretisierung erlauben. Es ist aber davon auszugehen, dass die Verteilung des Phyto- und Zooplanktons in einem See mit den Abmessungen des Bodensees aufgrund innerer und äußerer Faktoren auch horizontal starke Heterogenitäten aufweist. Das zeigen auch die Ergebnisse erster ökologischer 3-D-Modellierungen mit ELCOM-CAEDYM [1], [2] im Rahmen von BodenseeOnline.

Daher wurde im Mai 2007 eine seeweite Bodensee-Messkampagne durchgeführt. Ziel des Messprogramms war es, die räumliche Verteilung und kurzzeitige Dynamik physikalischer und biologischer Messgrößen, wie Wassertemperatur, Phyto- und Zooplankton, sowie der Fische zu erfassen.

Das Kooperationsnetzwerk Bodensee (KNB), bestehend aus der Landesanstalt

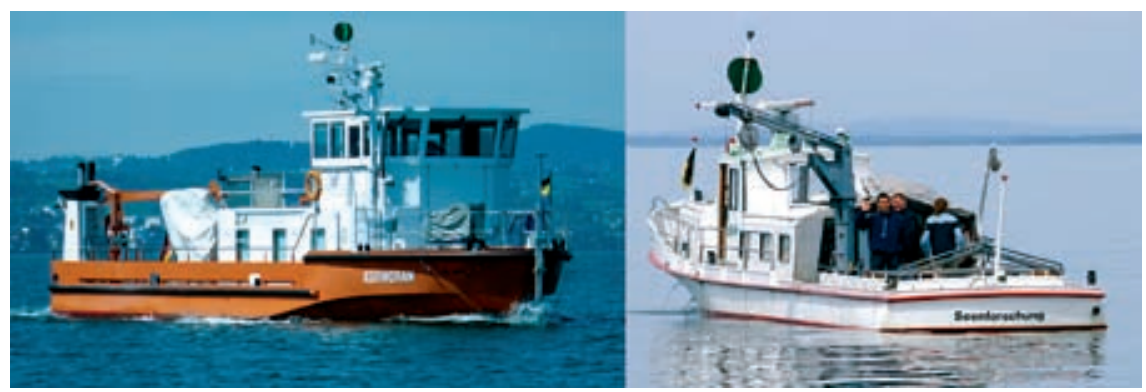

Bild 1: Zwei Forschungsschiffe des ISF im Einsatz (Bilder: Karsten Rinke, Christoph Krass)

für Umwelt, Messungen und Naturschutz Baden-Württemberg (LUBW) und den Universitäten Konstanz und Stuttgart, war der Initiator der Messkampagne. Konzeption und Organisation wurden im Rahmen des Forschungsverbundes BodenseeOnline realisiert.

\section{Methoden}

\subsection{Messtechnik}

Für die seeweite Beprobung mit Multiparametersonden waren vier Schiffe mit insgesamt drei Sonden, 4 hydroakustischen Messgeräten und 15 Personen zeitgleich im Einsatz. Bild 1 zeigt die zwei Forschungsschiffe des Instituts für Seenforschung der LUBW. Die insgesamt 168 Vertikalprofile von Temperatur und Chlorophyllkonzentration wurden mit Multiparametersonden aufgenommen, die mit Conductivity-Temperature-Depth-Sensoren (CTD-Sensoren) und Chlorophyllsensoren ausgestattet waren.

An zwei Tagen (10. und 16. Mai) wurde eine seeweite Erfassung realisiert (48 Positionen), während an anderen Messtagen

Konstanzer Online-Publikations-System (KOPS) einzelne Transekte beprobt wurden. Außerdem wurden an fünf verschiedenen Stellen am Ufer des Sees Thermistoren installiert, die in 1,5 m Wassertiefe kontinuierlich die Wassertemperatur aufzeichneten. Schließlich erfolgte eine kontinuierliche Erfassung der Wassertemperatur in verschiedenen Tiefen mittels einer Thermistorkette im Überlinger See.

Am Morgen des 8. Mai wurden des weiteren in der Friedrichshafener Bucht zur Bestimmung der oberflächennahen Strömungsverhältnisse drei Driftkörper ausgesetzt. Die Drifter bewegten sich auf gerundeten Bahnen Richtung Südosten und erreichten dabei Geschwindigkeiten von bis zu $0,21 \mathrm{~m} / \mathrm{s}$.

Die Verteilung von Zooplankton und Fischen wurde durch hydroakustische Methoden bestimmt. Das Zooplankton wurde mittels akustischer Rückstreuung mit einem ADCP (Acoustic Doppler Current Profiler) detektiert. Durch Beprobung und Auszählung des Zooplanktons wurde eine Korrelation zwischen der Zooplanktonabundanz und der akustischen Rückstreuung nachgewiesen. Allerdings geben die ADCP-Messungen keinerlei Aufschluss über Art und Größe der Organismen [3].

Ebenfalls hydroakustisch, jedoch mit Hilfe von Einzelechos statt Rückstreuungsintensität wurde auch die Fischverteilung erfasst.

\subsection{Meteorologische Bedingungen}

Der Zeitraum der Messkampagne war von sehr unterschiedlichen Windbedingungen geprägt. Während in der ersten Hälfte der Wind mit geringen Geschwindigkeiten aus wechselnden Richtungen 
wehte, kam am 8. Mai ein starker Wind aus westlicher Richtung auf, der bis zum 10. Mai mit Windgeschwindigkeiten von bis $\mathrm{zu} 10 \mathrm{~m} / \mathrm{s}$ und nahezu konstanter Windrichtung andauerte. Am Nachmittag des 10. Mai flaute der Wind wieder ab und kam in den nächsten Tagen, wie vor dem Sturm, aus wechselnden Richtungen, wobei die Windgeschwindigkeiten mit bis zu $6 \mathrm{~m} / \mathrm{s}$ etwas höher lagen als zuvor. Bild 2 zeigt die vom Deutschen Wetterdienst (DWD) in Konstanz gemessenen Windgeschwindigkeiten.

\section{Messergebnisse}

\subsection{Temperatur und Phytoplankton}

Das Bild 3 zeigt die gemessene Temperatur- und Chlorophyllverteilung in $5 \mathrm{~m}$ Tiefe am 10. (oben) und am 16. Mai (unten). Die Situation am 10. Mai ist vom vorhergehenden Windereignis geprägt: Der Sturm hat das warme Wasser des Epilimnions nach Osten gedrängt. Eine scharfe Front trennt kaltes, hypolimnisches Wasser mit geringen Chlorophyllkonzentrationen im Überlingersee und im Westteil des Hauptbeckens vom warmen, Phytoplankton-reichen Epilimnionwasser im Ostteil des Sees. Zum Zeitpunkt der Messung sind ca. $30 \%$ der Seeoberfläche von hypolimnischem Wasser bedeckt. Bei einer durchschnittlichen Tiefe der Thermokline von $15 \mathrm{~m}$ bedeutet das, dass etwa $6,4 \mathrm{~km}^{3}$ Epilimnionwasser über $25 \mathrm{~km}$ weit nach Osten bewegt worden sind. Die Temperatur variiert zwischen $6{ }^{\circ} \mathrm{C}$ und $14^{\circ} \mathrm{C}$. In dieser Situation ist der Einfluss der Hydrodynamik auf das Phytoplankton dominierend, es besteht eine positive Korrelation zwischen Wassertemperatur und Algenkonzentration in den obersten 15 Metern.

Ganz anders ist das Bild am 16. Mai: Zwar besteht der Temperaturgradient von Westen nach Osten weiterhin, die Differenzen sind jedoch wesentlich geringer. Da jetzt der starke äußere Einfluss des Sturms fehlt, besteht kaum eine Korrelation zwischen Wassertemperatur und Chlorophyllkonzentration mehr. Lediglich vor Meersburg ist eine Region mit besonders kaltem Wasser zu finden, in der auch die geringsten Konzentrationen an Chlorophyll gemessen wurden. Ansonsten ist die Verteilung der Algen nun stärker von internen Faktoren, wie Licht- und Nährstoffangebot, bestimmt. Die höchsten Konzentrationen befinden sich daher

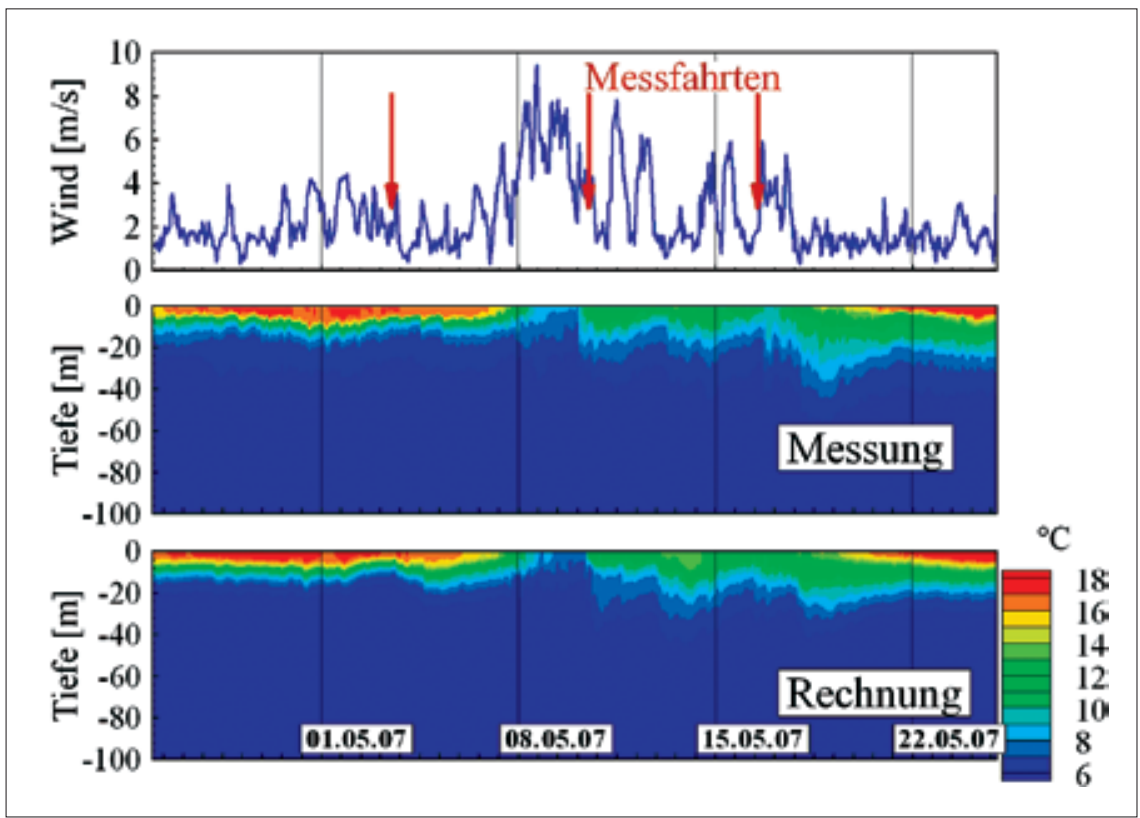

Bild 2: Windgeschwindigkeit (Konstanz DWD) im Zeitraum der Messkampagne (oben) und die hydrodynamische Reaktion des Sees: gemessenes (Mitte) und berechnetes (unten) Temperaturprofil an der Messboje der Universität Konstanz im Überlinger See nicht mehr in der Bregenzer Bucht, wo das Wasser am wärmsten ist, sondern im Bereich der alten Rheinmündung und im Westen des Überlinger Sees. Diese beiden seeweiten Erfassungen der Phytoplanktonverteilung demonstrieren die zwei grundlegenden Prozesse [4], die zu heterogenen Verteilungen (Patchiness) im Ökosystem führen: zum einen durch Transport und Verfrachtung der vorhandenen Biomasse (z. B. durch Wind), zum anderen durch unterschiedliche Wachstumsbedingungen im Habitat (z. B. durch lokale Eutrophierung).
Die Querschnitte in Bild 4 und 5 geben Aufschluss über die vertikale Verteilung (Transekt Fischbach-Uttwil). Die Algen halten sich vor dem Sturm bevorzugt im unteren Metalimnion auf.

\subsection{Hydroakustik hinsichtlich Zooplankton und Fischen}

In Bild 6 ist links die vom ADCP detektierte Rückstreustärke im Schnitt Fischbach-Uttwil aufgetragen. Hohe Vorkommen von Zooplankton sind nur im Epiund Metalimnion zu finden. Hier finden sie gute Wachstumsbedingungen in Form

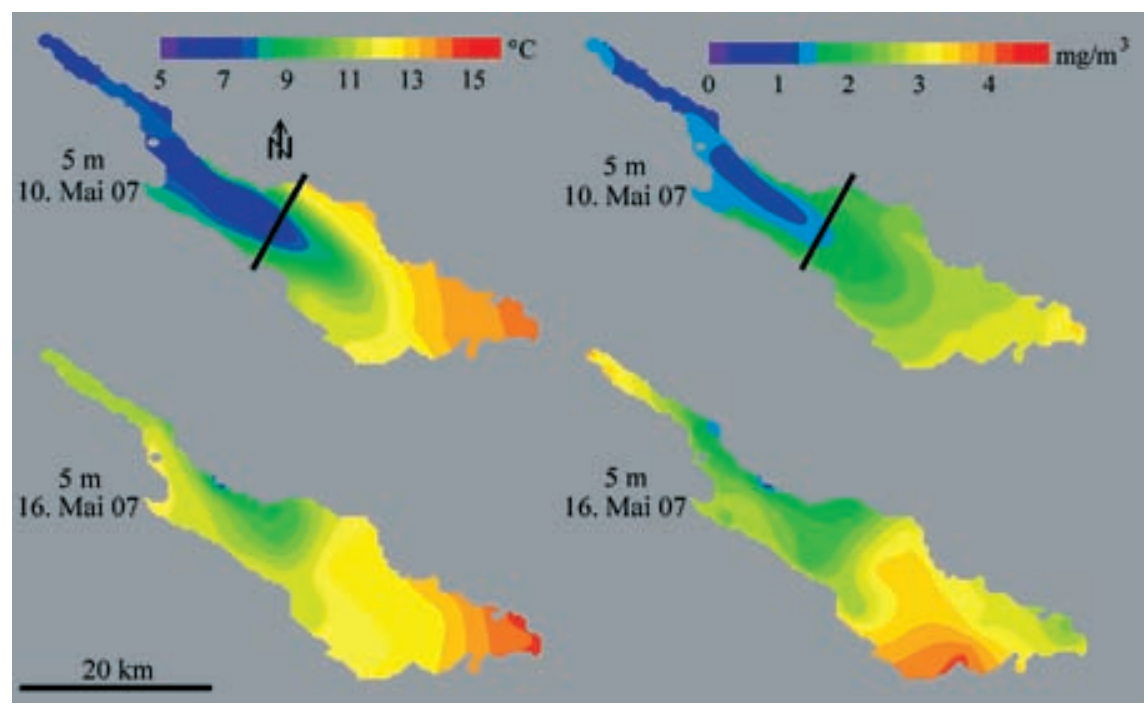

Bild 3: gemessene Temperatur- (links) und Chlorophyllverteilung (rechts) in $5 \mathrm{~m}$ Tiefe, die schwarzen Balken markieren den Querschnitt in den Bildern 4, 5 und 6 


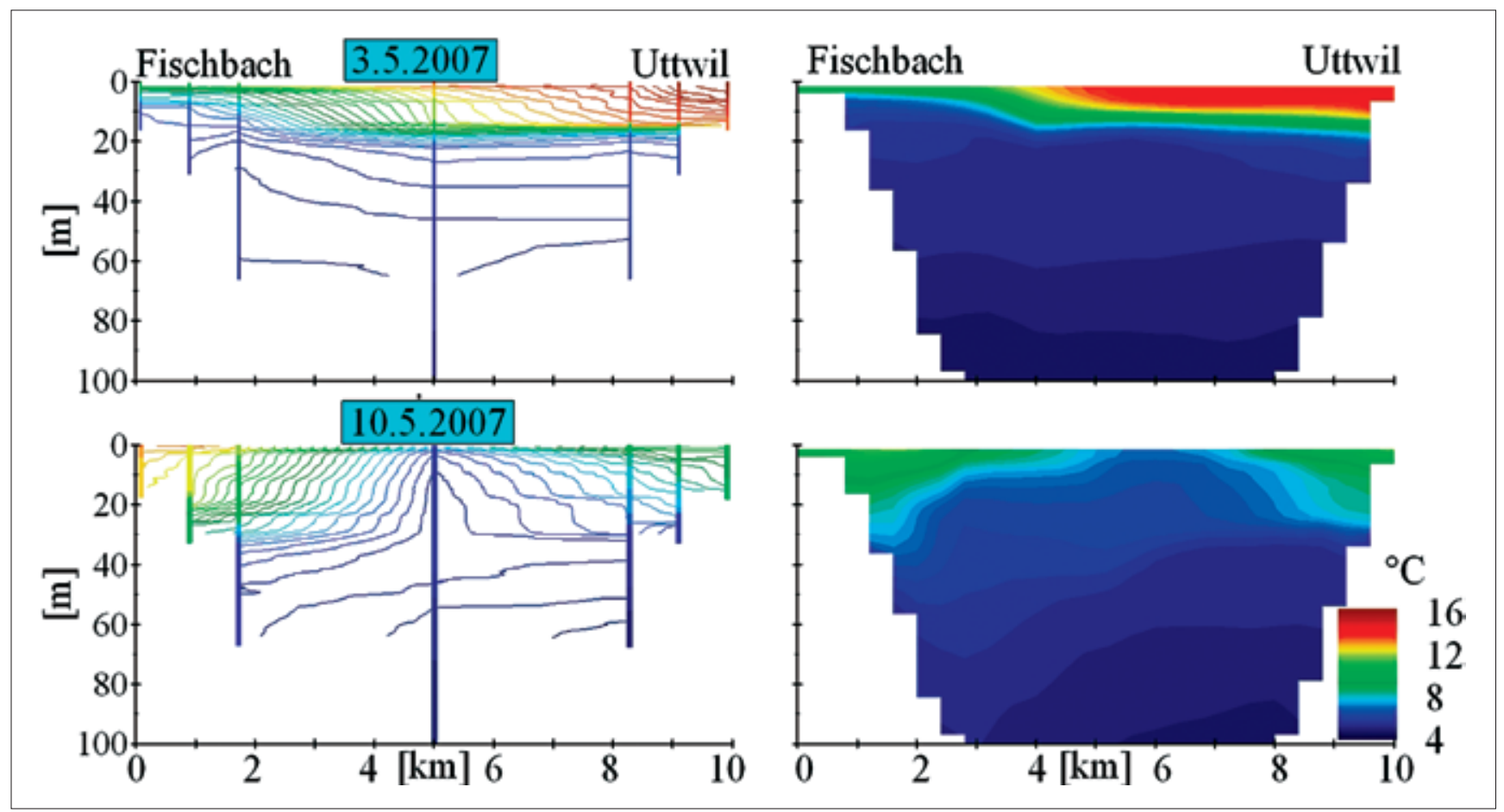

Bild 4: Temperaturverteilung im Querschnitt Fischbach-Uttwil, Vergleich Modell (rechts) -Messung (links)

von erhöhter Temperatur im Epilimnion und Algen als Nahrung (diese aber vor allem im unteren Metalimnion). Dabei fällt besonders am 3. Mai am Südufer auf, dass die Zooplankter die Schicht mit dem größten Temperaturgradient meiden und sich entweder im Epilimnion oder in einer dünnen Schicht im unteren Metalimnion aufhalten. Es ist davon auszugehen, dass die Tiere ihre Fähigkeit zur aktiven Bewegung nutzen und sich zeitweise im warmen, aber algenarmen Epilimnion aufhalten und zum Fressen nach unten in die vergleichsweise kalte Zone mit hohem Nahrungsangebot wandern.

Die hydroakustische Erfassung der Fische zeigte einen Gradienten entlang der Längsachse mit höheren Abundanzen im Ostteil des Sees, was sich mit vorherigen Untersuchungen deckt [4]. Hierbei ist aber zu beachten, dass die durchgeführten Messungen lediglich die Fischverteilung während des Tages erfassen konnten, welche von den Verteilungen in der Nacht deutlich abweichen können. Für detailliertere Informationen sei auf die Literatur [5] verwiesen. Obwohl Fische im Gegensatz zu planktischen Organismen durch ihre aktive Beweglichkeit in ihrer Verteilung weniger stark durch Strömungen beeinflusst werden, ist auch hier ein Einfluss des Sturms erkennbar. In den Bereichen mit kaltem Wasser im Transekt Fischbach-Uttwil wurden am 10. Mai we- niger Fische gefunden als in Bereichen mit warmem Wasser.

\section{Modelltechnische Auswertung der Messkampagne}

\subsection{Modellaufbau}

Zur Modellierung der Messkampagne wurde das ökologische Modell CAEDYM [2] gekoppelt mit dem hydrodynamischen 3-D-Modell ELCOM [1] verwendet. Beide Modelle wurden vom Centre of Water Research der University of Western Australia entwickelt.

Die horizontale Diskretisierung betrug $400 \mathrm{~m}$ x $400 \mathrm{~m}$ und vertikal wurde der See in 53 Tiefenschichten unterteilt, deren Mächtigkeit im Epi- und Metalimnion 2,5 $\mathrm{m}$ beträgt und dann schrittweise auf $10 \mathrm{~m}$ im tiefen Hypolimnion ansteigt.

Die Konfiguration der ökologischen $\mathrm{Pa}$ rameter wurde weitgehend vom bestehenden 1-D-Modell übernommen, das von Rinke et al. [6] in dieser Ausgabe der WasserWirtschaft beschrieben wird. Es wurden somit vier Phytoplanktongruppen und eine Zooplanktongruppe simuliert. Die vier Phytoplanktongruppen unterscheiden sich in Licht-, Phosphor und Siliziumbedarf sowie durch ihre Größe und stellen eine basale funktionelle Einteilung der im Bodensee zu unterschiedlichen Jahreszeiten auftretenden 154 Algentaxa dar.
Außerdem berechnet das Modell die Konzentration der Nährstoffe Stickstoff, Phosphor und Silizium sowie von Sauerstoff. Fische werden in der verwendeten Modellkonfiguration nur implizit als Mortalitätsfaktor des Zooplanktons berücksichtigt.

Als Anfangsbedingungen für Wassertemperatur und -inhaltsstoffe wurden Messdaten der Universität Konstanz vom 06.03.2007 verwendet, die meteorologischen Randbedingungen sind Messwerte des DWD der Stationen Konstanz und Bregenz.

Start der Simulation war der 6. März, womit das Modell vor Beginn der Messkampagne eine Vorlaufzeit von 2 Monaten hatte.

\subsection{Simulationsergebnisse Hydrodynamik}

Das 3-D-Modell simuliert die hydrodynamische Reaktion des Sees auf die antreibenden meteorologischen Faktoren. Die Simulationsergebnisse zeigen eine ausgezeichnete Übereinstimmung mit den im See gemessenen Wassertemperaturen (Bild 2). Beide zeigen die thermische Schichtung zu Beginn mit Oberflächentemperaturen um $16^{\circ} \mathrm{C}$. Am 10. Mai wird das Epilimnion nach Osten gedrängt, an der Messboje kommt hypolimnisches Wasser mit etwa 5 bis $6^{\circ} \mathrm{C}$ an die Oberfläche. Eineinhalb Tage später kehrt das Epilimnion wieder zurück. Es dauert je- 
doch noch fast zwei Wochen, bis die Oberflächentemperaturen wieder Werte erreichten, wie sie vor dem Sturm gemessen wurden. Auch der Vergleich der berechneten mit den gemessenen Verteilungen der Wassertemperatur im Querschnitt in Bild 4 zeigt, dass sowohl die internen Wellen in den Schwachwindphasen als auch die Reaktion auf das Starkwindereignis vom hydrodynamischen Modell gut wiedergegeben werden.

Am 3. Mai ist das Epilimnion im gezeigten Querschnitt am Schweizer Ufer deutlich mächtiger als am deutschen, auch die Oberflächentemperaturen sind hier höher als dort. Das ist das Ergebnis eines mäßigen Windes aus Nordosten, der am Nachmittag des 1. Mai aufkommt, die durch die interne Welle zu diesem Zeitpunkt bestehende Neigung der Thermokline in diese Richtung verstärkt, am Nachmittag des folgenden Tages auf Nordwest dreht und erst am Vormittag des 3. Mai so weit abflaut, dass das Epilimnion nach Norden zurückströmt.

Der Sturm aus Westsüdwest am 8. und 9. Mai verursacht eine deutliche, nach Osten gerichtete Strömung an der Seeoberfläche, die durch die Corioliskraft nach Süden abgelenkt wird und das Epilimnion nach Südosten drängt. Am 10. Mai lässt der Wind soweit nach, dass das warme Wasser beginnt, nach Westen zurückzuströmen, wobei es wieder durch die Cori- oliskraft nach rechts abgelenkt wird und deshalb am Nordufer entlang fließt. Dies führt dazu, dass im Querschnitt an beiden Ufern epilimnisches warmes Wasser zu finden ist, während in der Mitte noch das während des Sturms aus der Tiefe aufgestiegene kalte Wasser vorliegt (Bild 3).

\section{Phytoplankton}

Das Phytoplankton hält sich vor dem Windereignis im Modell wie in der Realität bevorzugt in der unteren Thermokline auf. Dies hängt vor allem mit dem Nährstoffangebot zusammen: Zu Beginn des Frühjahrs setzt das Algenwachstum oberflächennah ein, wo die Wachstumsbedingungen durch das gute Lichtangebot und die hohe Temperatur optimal sind. Mit der Zeit nimmt die Konzentration an verfügbarem Phosphor, dem limitierenden Nährstoff für die Algen im Bodensee, an der Oberfläche immer weiter ab, und das Maximum der Algenkonzentration ist in tieferen Schichten zu finden, wo die Phosphorkonzentration noch etwas höher ist. Vergleicht man die Verteilung der einzelnen Phytoplanktongruppen, so fällt auf, dass diejenigen mit einem höheren Phosphorbedarf früher in tiefere Schichten abwandern. Zusätzlich ist die vertikale Verteilung der Algen aber auch von deren Sinkgeschwindigkeit abhängig: Die kleineren Algen mit einer geringeren Sinkgeschwindigkeit sind weiter oben zu finden als die großen.
Die Verteilung der Algenkonzentration ist stark von der Hydrodynamik beeinflusst. Die Neigung der Thermokline am 3. Mai verlagert auch das Maximum der Algenkonzentration am Schweizer Ufer weiter nach unten. Der Sturm verdriftet mit den oberen Wasserschichten auch die Algen, die danach gemeinsam mit dem warmen Wasser zurückströmen. Das hypolimnische Wasser, das am 10. Mai in der Mitte des Transekts vorliegt, ist auch durch geringere Konzentrationen an Chlorophyll zu erkennen.

\section{Zooplankton}

Beim Vergleich der berechneten Zooplanktonkonzentrationen mit den Ergebnissen der hydroakustischen Messungen (Bild 6) ist zu berücksichtigen, dass erstere in $\mathrm{mg} / \mathrm{l}$ dargestellt sind, während bei letzteren die Rückstreustärke in dB angegeben ist, die in etwa proportional zur Konzentration ist. Direkte quantitative Vergleiche sind also nicht möglich, hier soll daher nur das Verteilungsmuster qualitativ verglichen werden.

Ebenso wie die Algen ist das Zooplankton durch die Hydrodynamik beeinflusst, allerdings tritt die höchste Konzentration in geringerer Tiefe auf. Im Modell besitzt das Zooplankton weder die Möglichkeit zur aktiven horizontalen oder vertikalen Fortbewegung noch eine Sinkgeschwindigkeit, so dass sich die Verteilung hier al-

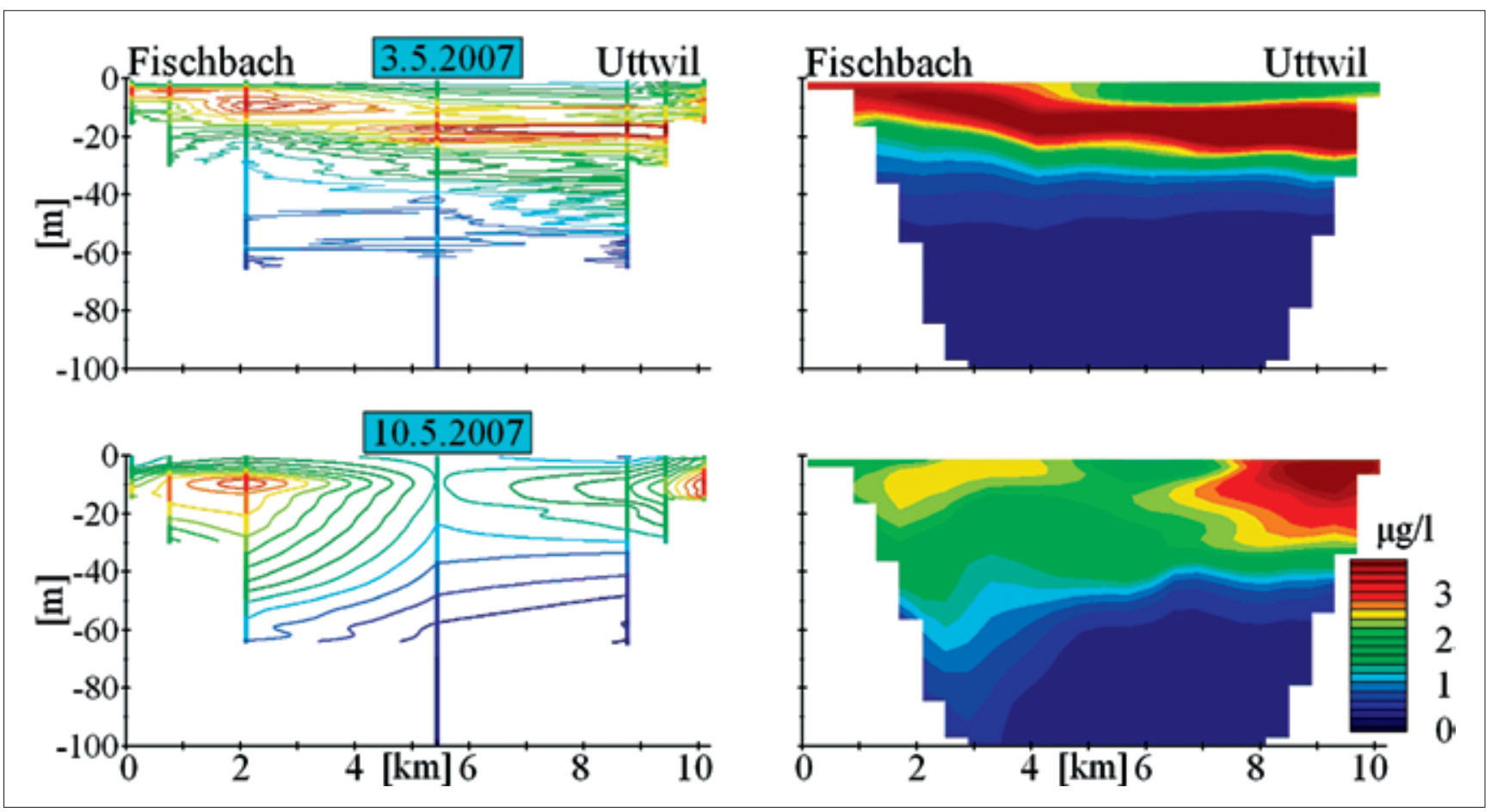

Bild 5: Chlorophyllverteilung im Querschnitt Fischbach-Uttwil, Vergleich Modell (rechts) -Messung (links) 


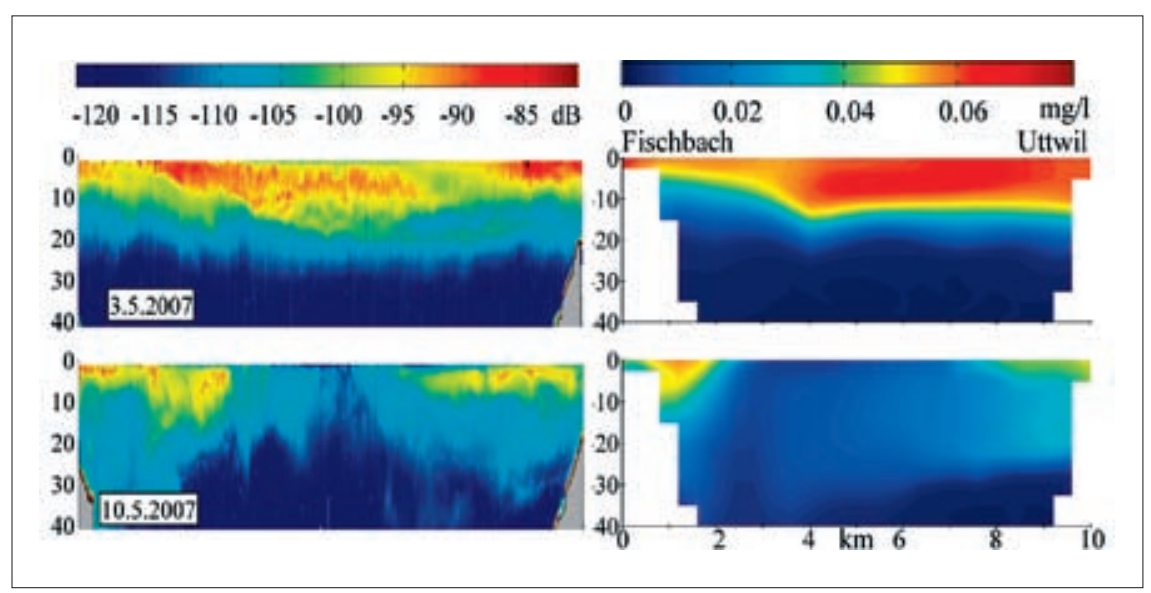

Bild 6: Zooplanktonverteilung im Querschnitt Fischbach-Uttwil, Vergleich Modell (rechts) -Messung (links)

lein aus den Wachstumsbedingungen, also Nahrungsangebot in Form von Phytoplankton und der Wassertemperatur, sowie der Verdriftung der Organismen mit der Seeströmung ergibt. Daher tritt die in der Messung beobachtete dünne Schicht hoher Abundanz knapp unterhalb der Thermokline im Modell nicht auf. Auch in flacheren Gewässern wurde gezeigt, dass interne Wellen und großskalige Strömungen die Zooplanktonverteilung beeinflussen [3].

\section{Zusammenfassung}

Die seeweite Messkampagne hat bestätigt, dass die horizontale Verteilung von Temperatur und Plankton im Bodensee stark variiert. Bei meteorologischen Extremereignissen, wie dem Sturm zwischen dem 8. und dem 10. Mai, dominiert der Einfluss der Hydrodynamik die Verteilung der Organismen, während bei ruhi- gerem Wetter andere Faktoren in den Vordergrund treten.

Das ökologische und hydrodynamische 3-D-Modell ELCOM-CAEDYM ist in der Lage, die Hydrodynamik und ihren Einfluss auf die Biologie gut wiederzugeben. Die Schwachwindsituation ist schwieriger zu reproduzieren. Vor allem die internen Einflüsse auf die Verteilung, wie Sukzession, Fraßdruck und Konkurrenz, werden von der verwendeten Modellkonfiguration noch unzureichend wiedergegeben. Um die Sukzession besser verfolgen und das Modell dementsprechend kalibrieren zu können, sind längere Messkampagnen nötig.

\section{Danksagung}

Die vorgestellten Arbeiten wurden von der DFG im Rahmen der Vorhaben Ko 528/19 und Ro 1008/11-1 gefördert. Die Autoren bedanken sich für die ihnen zuteil gewordene finanzielle Unterstützung. Wir danken den Schiffsbesatzungen für die Unterstützung bei den Messungen, Herrn F.
Magdalena Eder, Karsten Rinke, Sebastian Kempke, Andrea Huber and Thomas Wolf

\section{Lake-wide Field Campaign in Lake Constance 2007}

A lake-wide field campaign was conducted during spring 2007 in Lake Constance to assess three-dimensional distributions of temperature, phyto- and zooplankton and fish. The observed distributions of plankton and temperature were heavily affected by a strong westerly wind that forced the warm epilimnetic water into the eastern part of the lake. During that situation high abundances of plankton were present only in the eastern part of Lake Constance. At the same time cold, hypolimnetic water depleted of plankton was found at the surface in the western part. During low wind velocities phytoplankton distribution was governed by internal factors affecting plankton growth. For the period of the field campaign simulations were run with the coupled hydrodynamic-ecological Model ELCOM-CAEDYM, which showed a good agreement with the measurements, validating the applied model specification.
Peeters für die Bereitstellung von Daten der Thermistorkette im Überlinger See und Herrn R. Eckmann und N. Probst für die Auswertung der Echolotdaten.

\section{Autoren}

Dipl.-Ing. Magdalena Eder

Institut für Wasserbau

Universität Stuttgart

Pfaffenwaldring 61

70569 Stuttgart

Magdalena.Eder@iws.uni-stuttgart.de

\section{Dr. Karsten Rinke \\ Andrea Huber}

Limnologisches Institut

Universität Konstanz

Mainaustraße 252

78464 Konstanz

Karsten.Rinke@uni-konstanz.de

Andrea.Huber@uni-konstanz.de

\section{Dipl.-Ing. Sebastian Kempke}

Arbeitsgemeinschaft Wasserwerke

Bodensee-Rhein

Zweckverband Bodensee-Wasserversorgung

Süßenmühle 1

78354 Sipplingen

Sebastian.Kempke@zvbwv.de

\section{Dr. Thomas Wolf}

Institut für Seenforschung

Landesanstalt für Umwelt,

Messungen und Naturschutz

Baden-Württemberg

Argenweg 50/1

88085 Langenargen

Thomas.Wolf@lubw.bwl.de

\section{Literatur}

[1] Hodges, B.; Dallimore, C.: Estuary, Lake and Coastal Ocean Model: ELCOM. v2.2 Science Manual. Centre for Water Research, University of Western Australia, 2006.

[2] Hipsey, M. R.; Romero, J. R.; Antenucci, J. P.; Hamilton, D.: Computational Aquatic Ecosystem Dynamics Model: CAEDYM v2.3 Science Manual. Centre for Water Research, University of Western Australia, 2006.

[3] Rinke, K.; Hübner, I.; Petzoldt, T.; Rolinski, S.; Konig-Rinke, M.; Post, J.; Lorke, A.; Benndorf, J.: How internal waves influence the vertical distribution of zooplankton. In: Freshwater Biology 52 (2007), S.137-144.

[4] George, D. G.; Heaney, S. I.: Factors influencing the spatial distribution of phytoplankton in a small productive lake. In: Journal of Ecology 66 (1978), S. 133-155.

[5] Appenzeller, A.: Persistent large scale heterogeneity of pelagic fish in upper Lake Constance and its possible causes. In: Archiv für Hydrobiologie Special Issues Advances in Limnology 53 (1998), S. 303-316.

[6] Rinke, K.; Rothhaupt, K.: Ökologisches Modell des Bodensees: Konzept, Simulation und Test an Langzeitdaten. In: Wasserwirtschaft 98 (2008), Heft 10, S. 26-30. 